\title{
Entanglement negativity bounds for fermionic Gaussian states
}

\author{
Jens Eisert, ${ }^{1}$ Viktor Eisler, ${ }^{2,3}$ and Zoltán Zimborás ${ }^{1,4}$ \\ ${ }^{1}$ Dahlem Center for Complex Quantum Systems, Freie Universität Berlin, 14195 Berlin, Germany \\ ${ }^{2}$ Institute of Theoretical and Computational Physics, Graz University of Technology, Petersgasse 16, 8010 Graz, Austria \\ ${ }^{3}$ MTA-ELTE Theoretical Physics Research Group, Eötvös Loránd University, Pázmány Péter sétány 1/a, 1117 Budapest, Hungary \\ ${ }^{4}$ Department of Theoretical Physics, Wigner Research Centre for Physics, Hungarian Academy of Sciences, \\ H-1525 Budapest P.O. Box 49, Hungary
}

(Received 24 February 2018; published 13 April 2018)

\begin{abstract}
The entanglement negativity is a versatile measure of entanglement that has numerous applications in quantum information and in condensed matter theory. It can not only efficiently be computed in the Hilbert space dimension, but for noninteracting bosonic systems, one can compute the negativity efficiently in the number of modes. However, such an efficient computation does not carry over to the fermionic realm, the ultimate reason for this being that the partial transpose of a fermionic Gaussian state is no longer Gaussian. To provide a remedy for this state of affairs, in this work, we introduce efficiently computable and rigorous upper and lower bounds to the negativity, making use of techniques of semidefinite programming, building upon the Lagrangian formulation of fermionic linear optics, and exploiting suitable products of Gaussian operators. We discuss examples in quantum many-body theory and hint at applications in the study of topological properties at finite temperature.
\end{abstract}

DOI: 10.1103/PhysRevB.97.165123

\section{INTRODUCTION}

Entanglement is the distinct feature that makes quantum mechanics fundamentally different from a classical statistical theory. Undeniably playing a pivotal role in quantum information theory, in notions of key distribution, quantum computing and simulation, it is becoming clear that notions of entanglement have the potential to add a fresh perspective to the study of systems of condensed matter physics. Notions of entanglement entropies and spectra are increasingly used to capture properties of quantum systems with many degrees of freedom [1-3]. The entanglement entropy based on the vonNeumann entropy plays here presumably the most important role [1,2]. However, it makes sense as an entanglement measure only for pure states. Hence, early on, computable measures of entanglement such as the entanglement negativity [4-7] have been considered in the context of the study of quantum many-body systems. In fact, one of the earliest studies on entanglement properties of ground states of local Hamiltonians considered this entanglement measure [8], which was followed by a series of works on harmonic lattices [9-14].

Recent years have seen a revival of interest in studies of entanglement negativity, and the problem has been attacked using a number of different approaches. Numerical studies were performed for various spin chains via tensor network calculations [15-18], Monte Carlo simulations where the replica trick comes into play [19,20], or via numerical linked cluster expansion [21]. On the analytical side, major developments include the conformal field theory (CFT) approach [22,23], which has also been extended to finite temperature [24,25], nonequilibrium [24,26-28], and off-critical [29] scenarios. For some particular spin chains, there are even exact results available [30-33]. Studies of negativity have also been carried out for two-dimensional lattices [34,35] with a particular emphasis on topologically ordered phases [36-39].

The entanglement negativity-first proposed in Ref. [4], elaborated upon in Ref. [40], and proven to be an entanglement monotone in Refs. [5,6] — can be computed efficiently in the Hilbert space dimension for spin systems. For Gaussian bosonic systems, as they occur as ground and thermal states of noninteracting models, the negativity can even be efficiently computed in the number of modes $[6,8,41,42]$. This is possible because the partial transpose [43] on which the entanglement negativity is based, reflects partial time reversal [44], which maps bosonic Gaussian states to Gaussian operators. This is in sharp contrast to the situation for fermionic Gaussian systems, where the partial transpose is, in general, no longer a fermionic Gaussian operator [45]. Consequently, there is still no efficiently calculable formula known for the negativity. This is unfortunate, since Gaussian (or free) fermionic systems are specifically rich. For example, some well-known models showing features of topological properties such as Kitaev's honeycomb lattice model are noninteracting [46]. Also, one of the most paradigmatic one-dimensional models exhibiting edge states in a topologically nontrivial phase, the $\mathrm{Su}-\mathrm{Sch}$ rieffer-Heeger (SSH) model [47] is a noninteracting (or quasifree) fermionic system.

The lack of a formula for negativity of fermionic Gaussian states has stimulated a concerted research activity on identifying good bounds $[45,48]$. In this work, we make a fresh attempt at proving tight bounds to the entanglement negativity. Each bound considered here depends exclusively on the covariance matrix of the Gaussian state at hand, and thus is efficiently computable in the number of modes. In particular, the lower bound makes use of a pinching transformation of the covariance matrix, while the first of two upper bounds requires 
techniques of semidefinite programming. The second upper bound was already proposed in a CFT context [48], which is now elaborated and closed form expressions for arbitrary fermionic Gaussian states are given. We also test our bounds by estimating the negativity between adjacent segments in the $\mathrm{SSH}$ model and the XX chain, both in the ground state and at finite temperatures.

The paper is structured as follows. In Sec. II, we introduce the notation used in the rest of this work and define the negativity, followed by some basic examples given in Sec. III. The lower bound is constructed in Sec. IV, whereas Secs. V and VI deal with two different upper bounds, based on semidefinite programming and products of Gaussian operators, respectively. Numerical checks of the bounds are presented in Sec.VII, followed by our concluding remarks in Sec. VIII.

\section{PRELIMINARIES}

\section{A. Fermionic quantum systems}

Throughout this work, we consider quantum systems consisting of a set of fermionic modes; the annihilation and creation operators $\left\{f_{1}, f_{1}^{\dagger}, \ldots f_{k}, f_{k}^{\dagger}\right\}$ associated with the modes generates the CAR algebra, i.e., the algebra of operators respecting the canonical anticommutation relations. In many contexts, it is convenient to refer rather to Majorana fermions than to the original ones, by defining

$$
m_{2 j-1}=f_{j}^{\dagger}+f_{j}, \quad m_{2 j}=i\left(f_{j}^{\dagger}-f_{j}\right)
$$

for $j=1, \ldots, k$. Given a state $\rho$, the second moments of the Majorana fermions can be collected in the covariance matrix $\gamma \in \mathbb{R}^{2 k \times 2 k}$, with entries

$$
\gamma_{j, l}=\frac{i}{2} \operatorname{tr}\left(\rho\left[m_{j}, m_{l}\right]\right) .
$$

It is easy to see that this matrix satisfies

$$
\gamma=-\gamma^{T}, \quad i \gamma \leqslant \mathbb{1}
$$

We will denote the set of such covariance matrices of $k$ modes as $C_{k} \subset \mathbb{R}^{2 k \times 2 k}$.

A fermionic Gaussian state $\rho$ is completely defined by its covariance matrix, as one can express the expectation value of any Majorana monomial through the Wick expansion

$$
\operatorname{tr}\left(\rho m_{j_{1}} m_{j_{2}} \ldots m_{j_{2 p}}\right)=(-i)^{p} \sum_{\pi} \operatorname{sgn}(\pi) \prod_{l=1}^{p} \gamma_{j_{\pi(2 l-1)}, j_{\pi(2 l)}},
$$

where the indices of the Majorana operators are different and the sum runs over all pairings $\pi$ [with $\operatorname{sgn}(\pi)$ denoting the sign of the pairing].

Considering a Gaussian (or quasifree, as it is also called) unitary

$$
V=e^{-\frac{1}{4} \sum_{j, l} K_{j, l} m_{j} m_{l}}
$$

(where $K \in \mathbb{R}^{2 k \times 2 k}$ with $K=-K^{T}$ ) and a Gaussian state $\rho$, the evolved state $\rho^{\prime}=V \rho V^{\dagger}$ remains Gaussian. On the level of the covariance matrices, this mapping can be represented by the transformation

$$
\gamma \mapsto O_{K} \gamma O_{K}^{T}
$$

where $O_{K}=e^{-K} \in \mathrm{SO}(2 k)$. In this context, a commonly used tool is that a covariance matrix can be brought to a normal form by means of such a special orthogonal mode transformation $\widetilde{O}$,

$$
\widetilde{O} \gamma \widetilde{O}^{T}=\bigoplus_{j=1}^{k} x_{j}\left[\begin{array}{cc}
0 & -1 \\
1 & 0
\end{array}\right]
$$

with $x_{j} \in[-1,1]$ corresponding to the presence or absence of a fermion in the normal mode decomposition.

A Gaussian state is called particle-number conserving if it commutes with the particle-number operator $\sum_{j=1}^{k} f_{j}^{\dagger} f_{j}$. In this case, the expectation values of the pairing operators vanish, i.e., $\left\langle f_{j} f_{l}\right\rangle=\left\langle f_{j}^{\dagger} f_{l}^{\dagger}\right\rangle=0$. Thus the $2 k \times 2 k$ covariance matrix $\gamma$ can be completely recovered from the $k \times k$ correlation matrix $C_{j, l}=\left\langle f_{j}^{\dagger} f_{l}\right\rangle$. Moreover, such a state remains particle-number conserving and Gaussian under a mode transformations of the form $e^{-i \sum_{j, l} R_{j, l} f_{j}^{\dagger} f_{l}}$ (where $R$ is a Hermitian matrix), and the corresponding map on the correlation matrix level, analog of Eq. (6), is given by

$$
C \mapsto U_{R} C U_{R}^{\dagger}
$$

where $U_{R}=e^{-i R} \in U(k)$.

\section{B. Partial transpose and negativity}

Let us now turn to the definition of entanglement negativity. Consider a bipartite fermionic system composed of two subsystems $A$ and $B$ corresponding to Majorana modes $\left\{m_{1}, \ldots m_{2 n}\right\}$ and $\left\{m_{2 n+1}, \ldots m_{2 k}\right\}$, respectively. Following the literature, we will refer to such a setup as a bipartite system of $n \times(k-n)$ modes. Given a bipartite fermionic state $\rho$, the entanglement negativity is defined as

$$
\mathcal{N}=\frac{1}{2}\left(\left\|\rho^{T_{B}}\right\|_{1}-1\right)
$$

where $\|\cdot\|_{1}$ is the trace norm and the superscript $T_{B}$ denotes partial transposition with respect to subsystem $B$. The logarithmic negativity as a derived quantity is

$$
\mathcal{E}=\ln \left\|\rho^{T_{B}}\right\|_{1} .
$$

Both quantities have their significance, and the latter is an entanglement monotone despite not being convex [7], as well as an upper bound to the distillable entanglement. Since at the heart of the problem under consideration here is the assessment of $\left\|\rho^{T_{B}}\right\|_{1}$, a bound to the latter gives immediately a bound to both the negativity and the logarithmic negativity.

To proceed, we first need to represent the action of the partial transposition on the density operator. Using the notations $m_{j}^{0}=\mathbb{1}$ and $m_{j}^{1}=m_{j}$, a fermionic state can be written as

$$
\rho=\sum_{\tau} w_{\tau} m_{1}^{\tau_{1}} \ldots m_{2 k}^{\tau_{2 k}}
$$

where the summation runs over all bit-strings $\tau=$ $\left(\tau_{1}, \ldots, \tau_{2 k}\right) \in\{0,1\}^{\times 2 k}$ of length $2 k .^{1}$ The partial transpose of

\footnotetext{
${ }^{1}$ Note that a physical fermionic state must also commute with the parity operator $P=\prod_{j=1}^{2 k} m_{j}$, i.e., one has $w_{\tau}=0$ when $\sum_{j=1}^{2 k} \tau_{j}$ is odd.
} 
$\rho$ with respect to subsystem $B$ is the transformation that leaves the $A$ Majorana modes invariant and acts as a transposition $\mathcal{R}$ on the operators built up from modes of $B$, i.e.,

$$
\rho^{T_{B}}=\sum_{\tau} w_{\tau} m_{1}^{\tau_{1}} \ldots m_{2 n}^{\tau_{2 n}} \mathcal{R}\left(m_{2 n+1}^{\tau_{2 n+1}} \ldots m_{2 k}^{\tau_{2 k}}\right) .
$$

As shown in Ref. [45], the action of $\mathcal{R}$ in a suitable basis can be written as

$$
\mathcal{R}\left(m_{2 n+1}^{\tau_{2 n+1}} \ldots m_{2 k}^{\tau_{2 k}}\right)=(-1)^{f(\tau)} m_{2 n+1}^{\tau_{2 n+1}} \ldots m_{2 k}^{\tau_{2 k}},
$$

where

$$
f(\tau)= \begin{cases}0 & \text { if } \sum_{j=2 n+1}^{2 k} \tau_{j} \bmod 4 \in\{0,1\}, \\ 1 & \text { if } \sum_{j=2 n+1}^{2 k} \tau_{j} \bmod 4 \in\{2,3\} .\end{cases}
$$

As a main consequence one finds that, in sharp contrast to their bosonic counterparts, the partial transpose operation for fermionic Gaussian states does not preserve Gaussianity. Nonetheless, in a suitable basis the partial transpose can still be decomposed as the linear combination of two Gaussian operators [45].

\section{BASIC INSTANCES}

When discussing the negativity of Gaussian states, the situation of two fermionic modes is particularly instructive and and will be made use of later extensively. We hence treat this case in significant detail.

Any two-mode covariance matrix can be brought into the form

$$
\gamma=\left[\begin{array}{cccc}
0 & a & 0 & -b \\
-a & 0 & -c & 0 \\
0 & c & 0 & d \\
b & 0 & -d & 0
\end{array}\right]
$$

referred to as normal form, upon conjugating with $O_{A} \oplus O_{B}$, with $O_{A}, O_{B} \in S O(2)$, reflecting a local mode transformation in subsystems labeled $A$ and $B$. Such local mode transformation does not change the entanglement content of the state, and for a Gaussian state with a covariance matrix given by Eq. (15), one can easily compute the negativity. This is possible because one can identify the two-qubit system that reflects this Gaussian state by virtue of the Jordan-Wigner transformation. This two-qubit quantum state is given by the following expression.

Lemma 1. Negativity of two modes. Let $\gamma \in C_{2}$ be a covariance matrix in normal form. The negativity of the quantum state is that of the state

$$
\rho=\frac{1}{4}+\frac{1}{4}\left[\begin{array}{cccc}
M_{1,1} & 0 & 0 & M_{1,4} \\
0 & M_{2,2} & M_{2,3} & 0 \\
0 & M_{3,2} & M_{3,3} & 0 \\
M_{4,1} & 0 & 0 & M_{4,4}
\end{array}\right],
$$

of two qubits, where

$$
\begin{aligned}
& M_{1,1}=-(a+d)+(a d+b c), \\
& M_{2,2}=(a-d)-(a d+b c), \\
& M_{3,3}=-(a-d)-(a d+b c), \\
& M_{4,4}=(a+d)+(a d+b c), \\
& M_{1,4}=M_{4,1}=b+c, \\
& M_{2,3}=M_{3,2}=b-c .
\end{aligned}
$$

Hence the negativity of this state can be computed in closed form solving a simple quadratic problem. It is given by

$$
\mathcal{N}=\frac{1}{2}\left(\left\|\rho^{T_{B}}\right\|_{1}-1\right)=\frac{1}{2}(h(\gamma)-1),
$$

where we defined the function

$$
\begin{gathered}
h(\gamma)=\frac{1}{2}+\frac{1}{2} \max \left\{1, \sqrt{(a+d)^{2}+(b-c)^{2}}-(a d+b c),\right. \\
\left.\sqrt{(a-d)^{2}+(b+c)^{2}}+(a d+b c)\right\} .
\end{gathered}
$$

\section{A. Fermionic Gaussian pure-state entanglement}

A Gaussian state is pure if $\gamma^{2}=-\mathbb{1}$. In a $1 \times 1$ setup, this implies that by conjugating $\gamma$ with a local mode transformation $O_{A} \oplus O_{B}$ [where $\left.O_{A}, O_{B} \in \mathrm{SO}(2)\right]$, one can bring it into a Bardeen-Cooper-Schrieffer (BCS) form

$$
\gamma(a)=\left[\begin{array}{cccc}
0 & a & 0 & -b \\
-a & 0 & -b & 0 \\
0 & b & 0 & a \\
b & 0 & -a & 0
\end{array}\right]
$$

with $b:=\left(1-a^{2}\right)^{1 / 2}$. Thus the state depends on a single parameter $a \in[-1,1]$, and its negativity is given by

$$
\mathcal{N}=\frac{1}{2}\left(\left\|\rho^{T_{B}}\right\|_{1}-1\right)=\frac{1}{2}(g(a)-1),
$$

where we defined

$$
g(a)=1+\sqrt{1-a^{2}} .
$$

For a multimode fermionic Gaussian pure state, this gives rise to an explicit simple expression for the negativity, which we state in the following lemma.

Lemma 2. Pure fermionic Gaussian states. The negativity of a pure fermionic Gaussian state of $n \times n$ modes is

$$
\mathcal{N}=\frac{1}{2}\left(\prod_{j=1}^{n} g\left(a_{j}\right)-1\right)
$$

where $\left\{ \pm i a_{j}\right\}$ is the spectrum of $\gamma_{A}$.

Proof. It is known that any covariance matrix satisfying $\gamma^{2}=-\mathbb{1}$ can be brought into a multimode BCS form [49]:

$$
\begin{aligned}
& \left(O_{A} \oplus O_{B}\right) \gamma\left(O_{A} \oplus O_{B}\right)^{T}=\widetilde{\oplus}_{j=1}^{n} \gamma\left(a_{j}\right) \\
= & {\left[\begin{array}{cc}
\bigoplus_{j=1}^{n}\left[\begin{array}{cc}
0 & a_{j} \\
-a_{j} & 0
\end{array}\right] & \bigoplus_{j=1}^{n}\left[\begin{array}{cc}
0 & -b_{j} \\
-b_{j} & 0
\end{array}\right] \\
\hline \bigoplus_{j=1}^{n}\left[\begin{array}{cc}
0 & b_{j} \\
b_{j} & 0
\end{array}\right] & \bigoplus_{j=1}^{n}\left[\begin{array}{cc}
0 & a_{j} \\
-a_{j} & 0
\end{array}\right]
\end{array}\right], }
\end{aligned}
$$

where $\widetilde{\oplus}$ denotes a direct sum giving the above type of block structure, $O_{A}, O_{B} \in S O(2 n),\left\{ \pm i a_{j}\right\}$ is the spectrum of $\gamma_{A}$, and $a_{j}^{2}+b_{j}^{2}=1$. In other words, one can decouple the modes in $A$ and $B$ such that there is entanglement only between the corresponding pairs. Thus we can write (after rearranging the modes) the state as a product of these pairwise entangled $1 \times$ 1 -mode states. Using the multiplicativity of the trace norm and the negativity formulas Eqs. (21) and (22) for each of the decoupled $1 \times 1$ mode pairs, we arrive immediately at Eq. (23).

Let us also note that as for general pure states $\rho$,

$$
\left\|\rho^{T_{B}}\right\|_{1}=\operatorname{tr}\left(\rho_{A}^{1 / 2}\right)^{2}
$$


holds true, the negativity could anyway efficiently be computed via standard formulas for Rényi entropies of Gaussian states [50,51], yielding the same formula as Eq. (23).

For the sake of completeness, we mention that one can generalize the above results for any Gaussian state that can be brought by a local mode transformation into a state with the following type of covariance matrix:

$$
\left[\begin{array}{c|c}
\bigoplus_{j=1}^{n}\left[\begin{array}{cc}
0 & a_{j} \\
-a_{j} & 0
\end{array}\right] & \bigoplus_{j=1}^{n}\left[\begin{array}{cc}
0 & -b_{j} \\
-c_{j} & 0
\end{array}\right] \\
\hline \bigoplus_{j=1}^{n}\left[\begin{array}{cc}
0 & c_{j} \\
b_{j} & 0
\end{array}\right] & \bigoplus_{j=1}^{n}\left[\begin{array}{cc}
0 & d_{j} \\
-d_{j} & 0
\end{array}\right]
\end{array}\right]
$$

For states with such properties (e.g., for the isotropic states [49]), the negativity can be calculated using the general twomode formula Eq. (18), the final result being

$$
\mathcal{N}=\frac{1}{2}\left(\prod_{j=1}^{n} h\left(\gamma_{j}\right)-1\right),
$$

where $h\left(\gamma_{j}\right)$ is defined as in Eq. (19) with the corresponding parameters $a_{j}, b_{j}, c_{j}, d_{j}$.

\section{LOWER BOUND}

We now turn to presenting bounds to the entanglement negativity for arbitrary fermionic Gaussian states. We first discuss a lower bound, before proceeding to the more sophisticated upper bounds. The lower bound will be derived from a pinching transformation using the expression of two-mode negativity reviewed in the previous section.

\section{A. Lower bound from pinching}

Using the pinching transformation, one can decouple the system into independent $1 \times 1$ modes, and use for each of these system the previously obtained expression for the negativity for the $1 \times 1$ case. In the obtained expression, $\pi_{j}$ denotes the $4 \times 4$-submatrix associated with the respective $j$ th $1 \times 1$ subsystems.

Theorem 3. Lower bound. An efficiently computable lower bound of the negativity of a fermionic Gaussian state $\rho$ of $n \times n$ modes with covariance matrix $\gamma$ is for every $O_{A}, O_{B} \in \mathrm{SO}(2 n)$ provided by

$$
\mathcal{N}(\rho) \geqslant \frac{1}{2}\left(\prod_{j=1}^{n} h\left(\pi_{j}\left(O_{A} \oplus O_{B} \gamma O_{A}^{T} \oplus O_{B}^{T}\right)\right)-1\right) .
$$

Proof. In particular, $O_{A}=O_{B}=\mathbb{1}$ is a legitimate choice in this bound. The above statement follows from the fact that making use of random phases, one can group twirl the conjugate covariance matrix $\Gamma:=O_{A} \oplus O_{B} \gamma O_{A}^{T} \oplus O_{B}^{T}$ into

$$
\Gamma^{\prime}:=\widetilde{\bigoplus}_{j=1}^{n} \pi_{j}(\Gamma),
$$

for which the negativity can be readily computed as stated above. The group twirl amounts to a map

$$
\Gamma \mapsto \Gamma^{\prime}=\frac{1}{n} \sum_{j=1}^{n} O_{j} \Gamma O_{j}^{T}
$$

on the level of covariance matrices, where

$$
O_{j}:=\operatorname{diag}\left(H_{j}\right) \otimes \mathbb{1}_{4} .
$$

In this expression, $H_{j}, j=1, \ldots, n$, is the $j$ th row of a real Hadamard matrix

$$
H \in\{-1,1\}^{n \times n} \in O(n),
$$

an orthogonal matrix with \pm 1 entries. This is to show that the blocks of four Majorana operators are each equipped with signs, so that the resulting covariance matrix has the desired pinched form. The above group twirl can be performed with local operations and classical communication, hence it provides a lower bound, making use of the fact that the negativity is an entanglement monotone.

By choosing appropriate $O_{A}$ and $O_{B}$ (e.g., through an optimization procedure), one may obtain useful bounds for the entanglement negativity. The case of particle-number conserving Gaussian states is especially tractable.

\section{B. The particle number conserving case}

As discussed in Sec. II, when treating particle-number conserving Gaussian states, instead of the covariance matrix $\gamma$, we can work with the correlation matrix $C_{j, l}=\left\langle f_{j}^{\dagger} f_{l}\right\rangle$. When $C$ is real, one has the very simple relation

$$
\gamma_{2 j-1,2 l}=-\gamma_{2 l, 2 j-1}=2 C_{j, l}-\delta_{j, l},
$$

with all the other entries of $\gamma$ being zero.

Considering an $n \times n$ setup, we can divide the total correlation matrix of a state $\rho_{A \cup B}$ with respect to the two subsystems:

$$
C=\left[\begin{array}{l|l}
C_{A, A} & C_{A, B} \\
\hline C_{B, A} & C_{B, B}
\end{array}\right],
$$

where $C_{A, A}$ and $C_{B, B}$ are Hermitian, and $C_{A, B}^{\dagger}=C_{B, A}$. Let us choose the particle-number conserving local mode transformation $U_{A} \oplus U_{B}$ such that $U_{A} C_{A, B} U_{B}^{\dagger}$ is a positive diagonal matrix, i.e., $U_{A}$ and $U_{B}^{\dagger}$ provide the singular value decomposition of $C_{A, B}$. Applying now a pinching transformation on the mode-rotated state, we obtain a Gaussian state $\rho_{A \cup B}^{\prime}$ for which

$$
2 C^{\prime}-\mathbb{1}=\left[\begin{array}{ccc|ccc}
a_{1} & & & c_{1} & & \\
& \ddots & & & \ddots & \\
& & a_{n} & & & c_{n} \\
& & & & & \\
\hline c_{1} & & & d_{1} & & \\
& \ddots & & & \ddots & \\
& & c_{n} & & & d_{n}
\end{array}\right],
$$

where the nondiagonal elements of the block matrices are all zero, and $a_{j}, d_{j}$, and $c_{j}$ denote the diagonal elements of the matrices $\left(2 U_{A} C_{A, A} U_{A}^{\dagger}-\mathbb{1}\right),\left(2 U_{B} C_{B, B} U_{B}^{\dagger}-\mathbb{1}\right)$, and $2 U_{A} C_{A, B} U_{B}^{\dagger}$, 
respectively. Now, using Theorem 3, we obtain the following lower bound for the negativity for the original Gaussian state $\rho_{A \cup B}$ :

$$
\mathcal{N}\left(\rho_{A \cup B}\right) \geqslant \frac{1}{2}\left(\prod_{j=1}^{n} h\left(\gamma_{j}\right)-1\right),
$$

where

$$
\gamma=\left[\begin{array}{cccc}
0 & a_{j} & 0 & c_{j} \\
-a_{j} & 0 & -c_{j} & 0 \\
0 & c_{j} & 0 & d_{j} \\
-c_{j} & 0 & -d_{j} & 0
\end{array}\right]
$$

In Sec. VII, we will use this procedure to numerically calculate lower bounds for the negativity in the ground and thermal states of various many-body systems.

\section{UPPER BOUND VIA CONVEX OPTIMIZATION}

We turn now to presenting the first of our two novel strategies to arrive at upper bounds. It is rooted in ideas of convex optimization and the structure theorem of Gaussian maps obtained from the Lagrangian formulation of fermionic linear optics. The basic idea of this bound is to make use of the fact that the negativity is an entanglement monotone, thus by means of local transformation the negativity cannot increase on average. In this way, an upper bound can be identified once one is in the position to identify those Gaussian root states from which the desired state can be prepared and for which negativity can be calculated easily. As it turns out, this gives rise to a problem that can be tackled with the machinery of convex optimization.

\section{A. Fermionic Gaussian maps}

The bound as such will require some preparation. We start by stating how fermionic Gaussian maps (i.e., completely positive maps that send Gaussian states to Gaussian states) act on the level of covariance matrices.

Theorem 4. Structure of fermionic Gaussian maps [52]. An arbitrary fermionic Gaussian operation acts on covariance matrices $\gamma \in C_{m}$ as

$$
\gamma \mapsto B\left(\gamma^{-1}+D\right)^{-1} B^{T}+A,
$$

where

$$
\Gamma:=\left[\begin{array}{cc}
A & B \\
-B^{T} & D
\end{array}\right] \in C_{2 m}
$$

is a fermionic covariance matrix on a doubled mode space.

We now turn to an observation that is helpful in this context: all outcomes in a selective fermionic Gaussian map are related with each other upon conjugating the input with a diagonal matrix $P$ from $P_{m}$, with

$$
P_{m}:=\left\{P=\bigoplus_{j=1}^{m} x_{i} \mathbb{1}_{2}, x_{i} \in\{-1,1\}\right\} .
$$

This feature mirrors a similar property in the Gaussian bosonic setting, where with an appropriate shift in phase space conditioned on the measurement outcome, an arbitrary Gaussian map can be made trace-preserving $[53,54]$.
Lemma 5. Selective fermionic Gaussian operations. For any selective fermionic Gaussian operation, one outcome being described by a map (38), the other measurement outcomes are reflected by covariance matrices of the form

$$
\gamma \mapsto B\left(P \gamma^{-1} P+D\right)^{-1} B^{T}+A,
$$

where $P \in P_{m}$.

Proof. This means that all outcomes of a selective fermionic Gaussian map are on the level of covariance matrices reflected by the same transformation, upon conjugating the input by a matrix $P \in P_{m}$. This can be seen by acknowledging the fact that any post-selected fermionic completely positive map can be written as a concatenation of a fermionic Gaussian channel, acting as

$$
\gamma \mapsto X \gamma X^{T}+Y
$$

with $Y=-Y^{T}, X X^{T} \leqslant \mathbb{1}$, and $i Y \leqslant \mathbb{1}-X X^{T}$, in addition to dilations

$$
\gamma \mapsto O\left(\gamma \oplus \gamma^{\prime}\right) O^{T},
$$

with $\gamma^{\prime} \in C_{k}, O \in S O(2(m+k))$, followed by a fermion number measurement on the additional $k$ modes. This follows from Ref. [52], mirroring the situation for bosonic post-selected Gaussian completely positive maps [53,54]. For different outcomes of that fermionic measurement, the above map is being replaced by $(\mathbb{1} \oplus P) \Gamma(\mathbb{1} \oplus P), P \in P_{m}$. This means that for different measurement outcomes, the map in Eq. (38) is being replaced by

$$
\gamma \mapsto B P\left(\gamma^{-1}+P D P\right)^{-1} P B^{T}+A .
$$

This is identical with

$$
\gamma \mapsto B\left(P \gamma^{-1} P+D\right)^{-1} B^{T}+A .
$$

This structure can be uplifted to the level of local fermionic Gaussian operations, which seems helpful in its own right. From the above characterisation of fermionic Gaussian maps, we immediately obtain the following statement:

Lemma 6. Local fermionic Gaussian operations. Each outcome of a selective local fermionic Gaussian operation on an $n \times n$ system gives rise to a covariance matrix of the form

$$
\gamma \mapsto B\left(P \gamma^{-1} P+D\right)^{-1} B^{T}+A,
$$

where $A, B, D \in \mathbb{R}^{4 n \times 4 n}$ are submatrices of a covariance matrix as in Eq. (39) with $m=2 n, P \in P_{2 n}$ and block-diagonal matrices (with respect to the $n \times n$ partition) $A=A_{1} \oplus A_{2}$, $B=B_{1} \oplus B_{2}, D=D_{1} \oplus D_{2}$.

\section{B. Upper bound}

We are now in the position to develop the idea for the upper bound. The basic idea is that we would like to identify a simple $\xi \in C_{2 n}$, constituted of blocks of $4 \times 4$ matrices that reflect entangled pairs of fermionic modes, such that

$$
\gamma=B\left(\xi^{-1}+D\right)^{-1} B^{T}+A
$$

reflecting a local fermionic Gaussian operation. Using the monotonicity of the negativity, this gives rise to a tight upper bound. Introducing the notation for a standard completely 
entangled BCS covariance matrix for two mode systems

$$
G:=\left[\begin{array}{rrrr}
0 & 0 & 0 & -1 \\
0 & 0 & -1 & 0 \\
0 & 1 & 0 & 0 \\
1 & 0 & 0 & 0
\end{array}\right],
$$

we will state our theorem concerning the upper bound.

Theorem 7. Upper bound for the negativity. An efficiently computable upper bound of the negativity of a fermionic Gaussian state $\rho$ of $n \times n$ modes with covariance matrix $\gamma$ can be obtained from the solution of the semidefinite problem

$$
\min v:=\sum_{j=1}^{n} v_{j}
$$

subject to

$$
\begin{array}{r}
v_{j}=\left|\operatorname{tr}\left(G \eta_{j}\right)\right|, \quad \eta=\widetilde{\oplus}_{j=1}^{n} \eta_{j}, \quad i \eta \geqslant \mathbb{1}, \\
\eta_{j}=-\left[\begin{array}{cccc}
0 & \alpha_{j} & 0 & -\beta_{j} \\
-\alpha_{j} & 0 & -\beta_{j} & 0 \\
0 & \beta_{j} & 0 & \alpha_{j} \\
\beta_{j} & 0 & -\alpha_{j} & 0
\end{array}\right], \\
i\left[\begin{array}{cc}
\gamma-A & B \\
-B^{T} & \eta+D
\end{array}\right] \geqslant 0, i\left[\begin{array}{cc}
A & B \\
-B^{T} & D
\end{array}\right] \leqslant \mathbb{1},
\end{array}
$$

where $A=A_{1} \oplus A_{2}, B=B_{1} \oplus B_{2}, D=D_{1} \oplus D_{2}$ are blockdiagonal matrices with respect to the $n \times n$ decomposition, and $\widetilde{\oplus}$ denotes a direct sum as defined in Eq. (24). Given the solution of the optimal $v_{j}$ values, the negativity can be bounded as

$$
\mathcal{N} \leqslant \frac{1}{2} \prod_{j=1}^{n}\left(1+v_{j} / 4\right)-\frac{1}{2} .
$$

Proof. The logic of this argument is that the entanglement content of the Gaussian state described by the covariance matrix $\xi$ must be larger than that of $\gamma$, invoking the fact that the negativity is an entanglement monotone [5,6]. We can build upon the above characterization of fermionic Gaussian maps. What is more, each other outcome is related to the above upon conjugating $\xi$ with a $P$ of the above form.

We start from a $\xi \in C_{2 n}$, constituted of blocks $\xi_{j}$ of $4 \times 4$ for $j=1, \ldots, n$. These covariance matrices are taken to be of the form

$$
\xi_{j}=\left[\begin{array}{cccc}
0 & a_{j} & 0 & -b_{j} \\
-a_{j} & 0 & -b_{j} & 0 \\
0 & b_{j} & 0 & a_{j} \\
b_{j} & 0 & -a_{j} & 0
\end{array}\right],
$$

with $a_{j}^{2}+b_{j}^{2} \leqslant 1$. If a local fermionic Gaussian operation can be found, then for some suitable $A=A_{1} \oplus A_{2}, B=B_{1} \oplus B_{2}$, $D=D_{1} \oplus D_{2}$, and a $P \in P_{2 n}$ one has

$$
i \gamma=i B\left(P \xi^{-1} P+D\right)^{-1} B^{T}+i A,
$$

which can be relaxed into an inequality:

$$
i \gamma \geqslant i B\left(P \xi^{-1} P+D\right)^{-1} B^{T}+i A .
$$

The inverse is hard to handle in this expression, which is why we continue to incorporate the inverse directly into the convex program. Defining $\eta:=\xi^{-1}$, the constraint $i \xi \leqslant \mathbb{1}$ becomes

$$
i \eta \geqslant \mathbb{1} \text {. }
$$

We can now make use of a Schur complement [55] to relate (56) to a positive semidefinite constraint: the validity of

$$
i\left[\begin{array}{cc}
\gamma-A & B \\
-B^{T} & P \eta P+D
\end{array}\right] \geqslant 0
$$

also implies the validity of (56). At this point, the relaxed constraints become

$$
\begin{aligned}
& i\left[\begin{array}{cc}
\gamma-A & B P \\
-P B^{T} & \eta+P D P
\end{array}\right] \geqslant 0, \quad i\left[\begin{array}{cc}
A & B \\
-B^{T} & D
\end{array}\right] \leqslant \mathbb{1} \\
& \eta=\widetilde{\bigoplus}_{j=1}^{n} \eta_{j}, \quad i \eta \geqslant \mathbb{1}, \quad \eta=-\eta^{T},
\end{aligned}
$$

where $A=A_{1} \oplus A_{2}, B=B_{1} \oplus B_{2}$, and $D=D_{1} \oplus D_{2}$. We can impose the explicit form

$$
\eta_{j}=-\left[\begin{array}{cccc}
0 & \alpha_{j} & 0 & -\beta_{j} \\
-\alpha_{j} & 0 & -\beta_{j} & 0 \\
0 & \beta_{j} & 0 & \alpha_{j} \\
\beta_{j} & 0 & -\alpha_{j} & 0
\end{array}\right],
$$

of the inverses with $\alpha_{j}, \beta_{j} \in \mathbb{R}$. The connection between the entries of $\xi_{j}$ and $\eta_{j}$ are simply $\alpha_{j}=a_{j}\left(a_{j}^{2}+b_{j}^{2}\right)^{-1}$, $\beta_{j}=b_{j}\left(a_{j}^{2}+b_{j}^{2}\right)^{-1}$. Finding the $\xi=\eta^{-1}$ covariance matrix belonging to the parent Gaussian state with minimal negativity cannot be directly cast into a convex problem. However, we can introduce a parameter $v=\sum_{j} v_{j}$, where $v_{j}=\left|\operatorname{tr}\left(G \eta_{j}\right)\right|=$ $4\left|\beta_{j}\right|$, whose minimization as a convex optimization provides a quite optimal parent state. Given the negativity formula (27) for a block-diagonal $\xi$, we obtain the following upper bound

$$
\mathcal{N} \leqslant \frac{1}{2} \prod_{j=1}^{n} h\left(\xi_{j}\right)-\frac{1}{2} \leqslant \frac{1}{2} \prod_{j=1}^{n}\left(1+v_{j} / 4\right)-\frac{1}{2},
$$

where we used that

$$
\begin{aligned}
h\left(\xi_{j}\right) & =\frac{1}{2} \max \left\{2, a_{j}^{2}+b_{j}^{2}+2\left|b_{j}\right|+1\right\} \\
& =\frac{1}{2} \max \left\{2,1+\left(a_{j}^{2}+b_{j}^{2}\right)\left[1+2\left|b_{j}\right|\left(a_{j}^{2}+b_{j}^{2}\right)^{-1}\right]\right\} \\
& \leqslant \frac{1}{2} \max \left\{2,2+2\left|b_{j}\right|\left(a_{j}^{2}+b_{j}^{2}\right)^{-1}\right\} \\
& =1+\left|b_{j}\right|\left(a_{j}^{2}+b_{j}^{2}\right)^{-1}=1+v_{j} / 4 .
\end{aligned}
$$

The final statement follows from the fact that the $P \in P_{2 n}$ has no significance in the bound, and hence we can optimize for $P \in \mathbb{1}_{4 n}$. This ends the argument.

\section{UPPER BOUND FROM PRODUCTS OF GAUSSIAN OPERATORS}

We now turn to a second upper bound to the entanglement negativity, which complements the previous one and that serves a quite different aim. It can again be efficiently computed and allows for bounding the entanglement negativity in large systems. We now consider a system of $n$ modes, where now the modes are separated into subsets $A$ and $B$. We no longer require $A$ and $B$ to have the same cardinality, but can also allow for arbitrary cuts into a system $A$ and its complement. 
For any such division, we can define the operators $O_{ \pm}$as the Gaussian operators-which do not necessarily reflect quantum states-that have the fermionic covariance matrix

$$
\gamma_{ \pm}=T_{B}^{ \pm} \gamma T_{B}^{ \pm}
$$

where

$$
T_{B}^{ \pm}=\bigoplus_{j \in A} \mathbb{1}_{2} \bigoplus_{j \in B}( \pm i) \mathbb{1}_{2}
$$

In other words, $O_{ \pm}$are defined as the Gaussian operators satisfying

$$
\frac{i}{2} \operatorname{tr}\left(O_{ \pm}\left[m_{j}, m_{k}\right]\right)=\left(\gamma_{ \pm}\right)_{j, k} .
$$

Using this definition, the partial transpose of a Gaussian state can be written in the form [45]

$$
\rho^{T_{B}}=\frac{1-i}{2} O_{+}+\frac{1+i}{2} O_{-} .
$$

The main difficulty in evaluating the trace norm of the partial transpose is that its constituent Gaussian operators $O_{+}$and $O_{-}$ do not commute in general, and thus one has no direct access to the spectrum of $\rho^{T_{B}}$. Nevertheless, the simple form of Eq. (66) allows one to apply a triangle inequality to bound the trace norm as [48]

$$
\left\|\rho^{T_{B}}\right\|_{1} \leqslant\left\|\frac{1-i}{2} O_{+}\right\|_{1}+\left\|\frac{1+i}{2} O_{-}\right\|_{1}=\sqrt{2}\left\|O_{+}\right\|_{1},
$$

where we have used that the two terms in the linear combination are Hermitian conjugates of each other, hence their trace norms are equal. This gives for the negativity

$$
\mathcal{N} \leqslant \frac{1}{2}\left(\sqrt{2} \operatorname{tr}\left(O_{+} O_{-}\right)^{1 / 2}-1\right),
$$

whereas the logarithmic negativity can be upper bounded as

$$
\mathcal{E} \leqslant \ln \operatorname{tr}\left(O_{+} O_{-}\right)^{1 / 2}+\ln \sqrt{2} .
$$

The main advantage of these upper bounds is that they involve only the product of Gaussian operators $\mathrm{O}_{+} \mathrm{O}_{-}$, which is itself Gaussian and the traces of its powers can be expressed via appropriate covariance matrix formulas. To arrive to these expressions, it is useful first to introduce the normalized Gaussian density operator

$$
\rho_{\times}=\frac{O_{+} O_{-}}{\operatorname{tr}\left(O_{+} O_{-}\right)},
$$

with corresponding covariance matrix $\gamma_{\times}$. The rules of multiplication are simplest to obtain by considering the exponential form of the various Gaussian operators:

$$
\frac{1}{Z_{\sigma}} \exp \left(\sum_{k, l}\left(W_{\sigma}\right)_{k, l} m_{k} m_{l} / 4\right),
$$

where the superscripts $\sigma=+,-$ and $\times$ refer to the corresponding operator $O_{+}, O_{-}$, and $\rho_{\times}$. The matrices in the exponent are related to the covariance matrices via

$$
i \tanh \frac{W_{\sigma}}{2}=\gamma_{\sigma}, \quad \exp \left(W_{\sigma}\right)=\frac{1-i \gamma_{\sigma}}{1+i \gamma_{\sigma}},
$$

and the normalization factors are given by

$$
Z_{\sigma}=\tilde{\operatorname{det}}\left(\mathbb{1}+\exp \left(W_{\sigma}\right)\right) \text {. }
$$

Here the symbol det denotes that the double degenerate eigenvalues of the corresponding matrix have to be counted only once, i.e., it is the square root of the determinant up to a possible sign factor. Using Eqs. (71) and (72), the solution for $\gamma_{\times}$can be found after simple algebra as [56]

$$
-i \gamma_{\times}=\mathbb{1}-\left(\mathbb{1}+i \gamma_{-}\right)\left(\mathbb{1}-\gamma_{+} \gamma_{-}\right)^{-1}\left(\mathbb{1}+i \gamma_{+}\right) .
$$

With the multiplication rule at hand, we are now ready to evaluate the trace norm

$$
\left\|O_{+}\right\|_{1}=\operatorname{tr}\left(O_{+} O_{-}\right)^{1 / 2}=\operatorname{tr}\left(\rho_{\times}\right)^{1 / 2}\left(\frac{Z_{\times}}{Z_{+} Z_{-}}\right)^{1 / 2}
$$

appearing in the upper bounds (68) and (69). Using (72) and (73), the ratio of the normalization factors can be rewritten as

$$
\frac{Z_{\times}}{Z_{+} Z_{-}}=\operatorname{det} \frac{\mathbb{1}-\gamma_{+} \gamma_{-}}{2}=\operatorname{det} \frac{\mathbb{1}-\gamma^{2}}{2} .
$$

For the other term, we can use the well-known trace formula for Gaussian states

$$
\operatorname{tr} \rho_{\times}^{\alpha}=\tilde{\operatorname{det}}\left[\left(\frac{\mathbb{1}+i \gamma_{\times}}{2}\right)^{\alpha}+\left(\frac{\mathbb{1}-i \gamma_{\times}}{2}\right)^{\alpha}\right],
$$

with $\alpha=1 / 2$. Hence the upper bounds can be calculated explicitly in terms of the covariance matrices $\gamma_{\times}$and $\gamma$.

Before moving to the study of concrete examples, let us comment about the spectral properties of $\gamma_{\times}$. By a similarity transformation one can permute the factors in the second term of (74) to arrive at

$$
\gamma_{\times} \simeq\left(\mathbb{1}-\gamma_{+} \gamma_{-}\right)^{-1}\left(\gamma_{+}+\gamma_{-}\right)
$$

where $\simeq$ denotes equivalence of the spectra. Furthermore, using the definition in Eq. (63), one can write

$$
\gamma_{\times} \simeq\left(\frac{\mathbb{1}-\gamma^{2}}{2}\right)^{-1} \frac{\gamma R+R \gamma}{2}
$$

where $R=\left(T_{B}^{+}\right)^{2}=\left(T_{B}^{-}\right)^{2}=\mathbb{1}_{2|A|} \oplus-\mathbb{1}_{2|B|}$. Thus the second term in (79) becomes block diagonal,

$$
\frac{\gamma R+R \gamma}{2}=\gamma_{A} \oplus-\gamma_{B},
$$

with the sign of the reduced covariance matrix of the $B$ modes being reversed. In particular, if the state on $A \cup B$ is pure, i.e., $\gamma^{2}=-\mathbb{1}$, then the spectrum of $\gamma_{\times}$is simply given by the eigenvalues of $\gamma_{A}$ and $-\gamma_{B}$, respectively. Moreover, since the spectrum of $\gamma_{A}$ and $\gamma_{B}$ are identical (up to trivial eigenvalues $\pm i$ if $|A| \neq|B|)$ this just leads to a double degeneracy.

For the upper bound of the logarithmic negativity, it is useful to define the quantity

$$
\hat{\mathcal{E}}=\ln \operatorname{tr}\left(O_{+} O_{-}\right)^{1 / 2}
$$

such that $\mathcal{E} \leqslant \hat{\mathcal{E}}+\ln \sqrt{2}$. Then using (75)-(77), $\hat{\mathcal{E}}$ can be expressed via Renyi entropies as

$$
\hat{\mathcal{E}}=\frac{1}{2}\left[S_{1 / 2}\left(\rho_{\times}\right)-S_{2}\left(\rho_{A \cup B}\right)\right],
$$

where for any state $\rho$,

$$
S_{\alpha}(\rho)=\frac{1}{1-\alpha} \ln \operatorname{tr} \rho^{\alpha} .
$$


In particular, for pure states one has $S_{2}\left(\rho_{A \cup B}\right)=0$, while $S_{1 / 2}\left(\rho_{\times}\right)=2 S_{1 / 2}\left(\rho_{A}\right)$ due to the double degeneracy of the $\gamma_{\times}$ spectrum mentioned above, and hence $\mathcal{E}=\hat{\mathcal{E}}=S_{1 / 2}\left(\rho_{A}\right)$. In other words, for pure states the upper bound is tight without the additional constant $\ln \sqrt{2}$, since the operators $O_{+}$and $O_{-}$ commute.

\section{NUMERICAL EXAMPLES}

In this section, we will test the covariance-matrix based bounds introduced before on the concrete example of a dimerized XX chain. After Jordan-Wigner transformation, this is equivalent to a noninteracting fermionic chain with an alternating hopping $t_{ \pm}=1 \pm \delta$, given by the Hamiltonian

$$
H=-\frac{1}{2} \sum_{j}\left(t_{+} f_{2 j}^{\dagger} f_{2 j-1}+t_{-} f_{2 j+1}^{\dagger} f_{2 j}+\text { H.c. }\right) \text {, }
$$

with dimerization parameter $-1 \leqslant \delta \leqslant 1$. This is also called the SSH chain. In all our examples, we consider an open chain with even sites $N$ at half-filling, and calculate the entanglement between the modes of two adjacent intervals, such that the spinand fermion-chain negativity are indeed equivalent.

A further simplification occurs due to the fact, that the Hamiltonian is particle-number conserving. On one hand, this allows us to implement our simple construction for the lower bound. On the other hand, it makes the calculations for the upper bound easier, since all the information is encoded in the fermionic correlation matrix elements $C_{m, n}=\left\langle f_{m}^{\dagger} f_{n}\right\rangle$. As already noted in [34], for a particle-conserving Gaussian state with real $C$ one can replace the covariance matrix $-i \gamma \mapsto G=$ $2 C-1$ and define the matrices $G_{ \pm}$and $G_{\times}$correspondingly. The formulas leading to the upper bound are then completely analogous to (76) and (77), except that the det symbols have to be replaced by ordinary determinants.

\section{A. Bounds versus exact results}

First, we test both lower and upper bounds against exact calculations of the logarithmic negativity for small chain sizes $N \leqslant 10$. For simplicity, we consider two adjacent intervals of the same size $\ell$, taken symmetrically from the center of the chain. We will consider both ground and thermal states of the dimerized chain, for which the fermionic correlation matrix elements read

$$
C_{m, n}=\sum_{k=1}^{N} \frac{\phi_{k}^{*}(m) \phi_{k}(n)}{e^{\beta \omega_{k}}+1},
$$

where $\omega_{k}$ and $\phi_{k}(m)$ are the single-particle eigenvalues and eigenvectors of the Hamiltonian (84).

Before presenting our data, let us comment on an observation about the upper bound. Although the inequality reads $\mathcal{E} \leqslant \hat{\mathcal{E}}+\ln \sqrt{2}$, in all our numerics we observe that the bound is actually tighter, i.e., one has $\breve{\mathcal{E}} \leqslant \mathcal{E} \leqslant \hat{\mathcal{E}}$. This has also been conjectured in Ref. [48] but a rigorous proof is lacking.

In our first example we consider the ground state of a chain with $N=8$ and $\ell=2$. The data are shown in Fig. 1 as a function of the dimerization parameter $\delta$. Note that, since $N / 2=4$ is even, the hopping between the two subsystems is given by $1-\delta$. Thus the entanglement vanishes for $\delta=1$

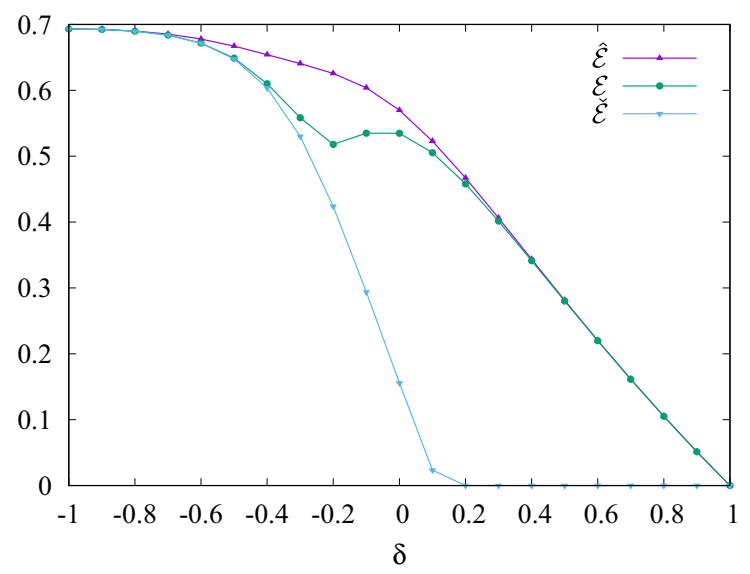

FIG. 1. Logarithmic negativity bounds vs exact results in the ground state, as a function of the dimerization $\delta$, with $N=8$ and $\ell=2$.

while it is given by $\ln 2$ at the other extreme $\delta=-1$, where a singlet is formed in the center. As expected from its construction, the lower bound $\breve{\mathcal{E}}$ performs well only in the region $\delta<0$, where one has a singlet-type dominant contribution to the entanglement. Remarkably, the upper bound $\hat{\mathcal{E}}$ gives an overall good performance on both sides, with an almost perfect saturation for $\delta>0.2$. However, approaching $\delta \rightarrow-1$, the entanglement tends to stay closer to its lower bound.

It is very instructive to have a look also at the thermal case. Here we consider the two halves of a chain with $N=8$ sites as subsystems and vary the temperature. This scenario exhibits a very rich physics, as depicted on Fig. 2, where now the symbols show the exact data, whereas the solid lines with matching colors give the respective bounds. In fact, in the regime $\delta<0$ where the couplings at the boundaries are weak, the Hamiltonian (84) supports edge states. Consequently, the ground state shows topological features which yields an additional $\ln 2$ contribution to the entanglement as $\delta \rightarrow-1$. Since the state is pure, one has $\mathcal{E}=\hat{\mathcal{E}}$, as discussed earlier.

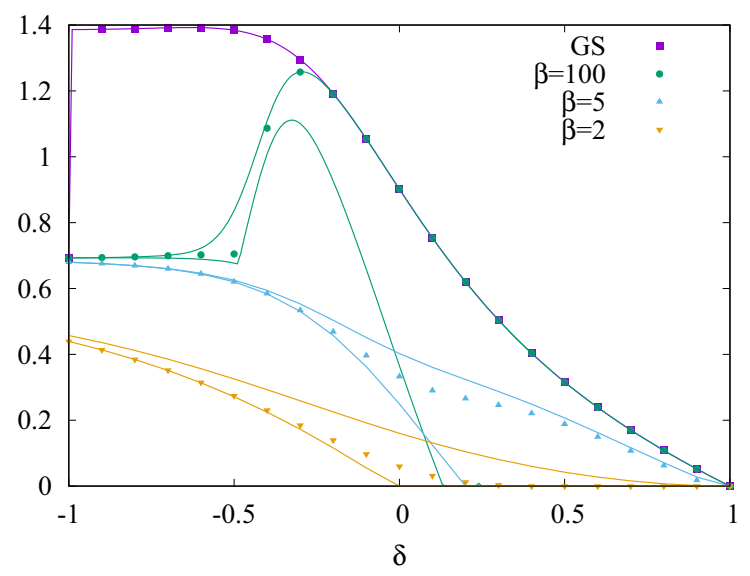

FIG. 2. Logarithmic negativity bounds vs exact results for thermal states, as a function of the dimerization $\delta$, and for various values of $\beta$. The symbols represent the exact data, while the solid lines with matching colors show the corresponding bounds. 
However, already a slight increase of the temperature (see $\beta=100$ ) seems to destroy this order, hence the topological contribution to the entanglement vanishes. Not surprisingly, for these low temperatures, the upper bound gives a very good overall estimation. Nevertheless, for increasing temperatures, the data gradually move towards the lower bound. This improved performance can be understood by a simple argument. The construction of the lower bound erases all the correlations within each subsystem $A$ and $B$. At higher temperatures, however, such correlations are already washed out and thus the approximation is more valid.

\section{B. Upper bound for infinite homogeneous chain}

From now on we focus on the homogeneous chain $\delta=0$, and take the thermodynamic limit $N \rightarrow \infty$. The Hamiltonian is then diagonalized by a Fourier transform and the correlation matrix takes the simple form

$$
C_{m, n}=\int_{-\pi}^{\pi} \frac{d q}{2 \pi} \frac{e^{-i(m-n)}}{e^{-\beta \cos q}+1} .
$$

Our main goal is to study the scaling of the upper bound as a function of the inverse temperature $\beta$ and subsystem sizes $|A|=\ell_{1}$ and $|B|=\ell_{2}$ and compare it to the predictions of CFT [22].

\section{Ground state}

We start with the study of $\hat{\mathcal{E}}$ in the ground state and take $\ell_{1}=\ell_{2}=\ell$ for simplicity. Invoking Eq. (82), one observes that the upper bound can be written as the difference of two Rényi entropies, with respect to Gaussian states $\rho_{A \cup B}$ and $\rho_{\times}$. Note that, while the former is just the reduced density operator of an interval of size $2 \ell$ in an infinite hopping chain, the latter one has no particular physical interpretation.

To understand the scaling behavior of the entropies, it is useful to have a look at the corresponding free-fermion entanglement Hamiltonians $\mathcal{H}$ and $\mathcal{H}_{\times}$, defined by [57]

$$
\rho_{A \cup B}=\frac{e^{-\mathcal{H}}}{\mathcal{Z}}, \quad \rho_{\times}=\frac{e^{-\mathcal{H}_{\times}}}{\mathcal{Z}_{\times}} .
$$

Their single-particle spectra, $\varepsilon_{k}$ and $\varepsilon_{k}^{\times}$, respectively, are related via

$$
\zeta_{k}=\tanh \frac{\varepsilon_{k}}{2}, \quad \zeta_{k}^{\times}=\tanh \frac{\varepsilon_{k}^{\times}}{2}
$$

to the spectra $\zeta_{k}$ of $G$ and $\zeta_{k}^{\times}$of $G_{\times}$. Owing to the simple thermal form (87) of the density operators, the calculation of Renyi entropies reduces to evaluating entropy formulas for a Fermi gas. In fact, the leading contributions to the entropies are delivered by the low-lying eigenvalues of the spectra. For the entanglement Hamiltonian $\mathcal{H}$, these were studied before and, for $\ln \ell \gg 1$, are given approximately by [58,59]

$$
\varepsilon_{k}=\frac{\pi^{2}(k-1 / 2-\ell)}{\ln (4 \ell)-\psi(1 / 2)},
$$

with the digamma function $\psi(1 / 2) \approx-1.963$. Thus the entanglement Hamiltonian has a level spacing inversely proportional to $\ln \ell$, or in other words, a logarithmic density of states. In turn,

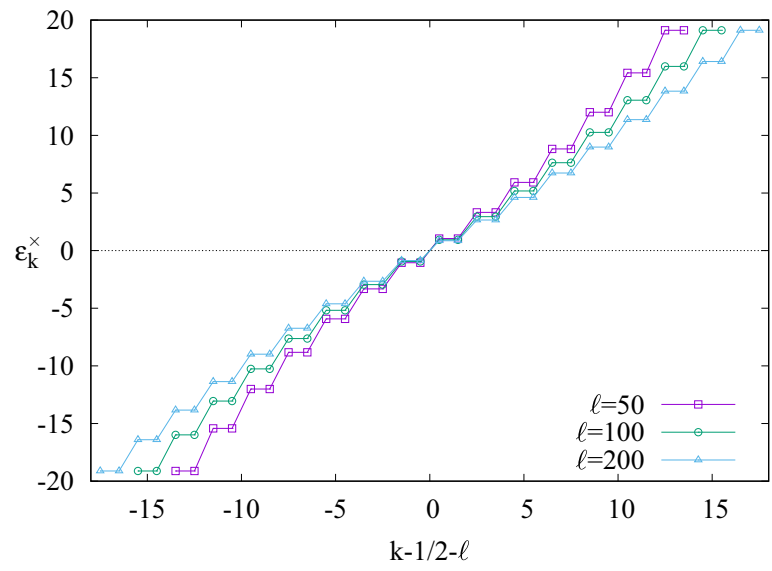

FIG. 3. Single-particle spectra $\varepsilon_{k}^{\times}$for various $\ell$.

this yields the celebrated result for the Renyi entropies

$$
S_{\alpha}\left(\rho_{A \cup B}\right)=\frac{1}{6}\left(1+\alpha^{-1}\right) \ln \ell+\text { const. }
$$

We shall now have a look at the $\operatorname{spectra} \varepsilon_{k}^{\times}$and their behavior as a function of $\ell$, shown in Fig. 3. Apart from the double degeneracy of the eigenvalues, the spectra show very similar features to those of $\varepsilon_{k}$. In particular, one can observe the slow logarithmic variation of the spacing and the approximate linear behavior around zero. We thus propose the ansatz

$$
\varepsilon_{2 k-1}^{\times}=\varepsilon_{2 k}^{\times}=a \frac{\pi^{2}(k-1 / 2-\ell / 2)}{\ln (2 \ell)+b},
$$

with fitting parameters $a$ and $b$. Fitting the lowest-lying eigenvalue as a function of $\ell$, we obtain $a=1.325 \approx 4 / 3$ and $b=1.655$ where, for better fit results, we also included a subleading term proportional to $1 / \ell$. Note that the higher part of the spectrum shows a slight upward bend, which is again very similar to the behavior of the $\varepsilon_{k}$ spectra [59].

From the ansatz in Eq. (91) it is very easy to infer the leading scaling behavior of the Renyi entropies. Indeed, the main difference from (89) is the increased level spacing, leading to a decrease of the density of states by a factor of $a^{-1} \approx 3 / 4$. Taking into account also the double degeneracy of the spectrum, one arrives at

$$
S_{\alpha}\left(\rho_{\times}\right)=\frac{1}{4}\left(1+\alpha^{-1}\right) \ln \ell+\text { const. }
$$

That is, ignoring the subleading constant, which is also modified due to the parameter $b$, the entropies $S_{\alpha}\left(\rho_{\times}\right)$and $S_{\alpha}\left(\rho_{A \cup B}\right)$ differ by a factor of $3 / 2$. This is indeed the result we find numerically by fitting the data for various $\alpha$. Finally, inserting the appropriate Renyi entropies into (82), one immediately finds

$$
\hat{\mathcal{E}}=\frac{1}{4} \ln \ell+\text { const. }
$$

Thus the upper bound shows exactly the same scaling as the logarithmic negativity predicted by CFT calculations with central charge $c=1$ [22].

It is instructive to have a look also at the case of unequal adjacent segments of size $\ell_{1}$ and $\ell_{2}$, where the CFT prediction 


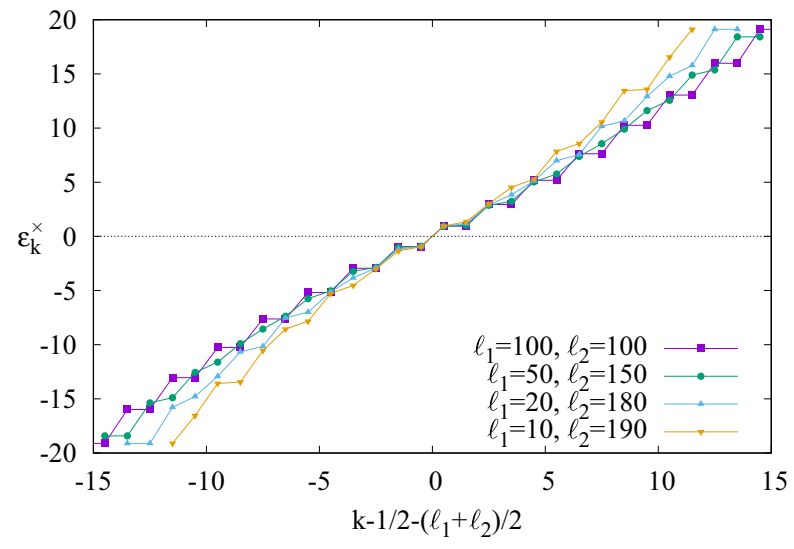
$\ell_{1}$.

FIG. 4. Single-particle spectra $\varepsilon_{k}^{\times}$for $\ell_{1}+\ell_{2}=200$ and various

gives [22]

$$
\mathcal{E}=\frac{c}{4} \ln \frac{\ell_{1} \ell_{2}}{\ell_{1}+\ell_{2}}+\text { const. }
$$

The corresponding spectra $\varepsilon_{k}^{\times}$are shown in Fig. 4, for a fixed overall length $\ell_{1}+\ell_{2}=200$ and varying $\ell_{1}$. The main feature to be seen is the breaking of the degeneracies. Indeed, from the analog of Eq. (79) to the present case, it is clear that the spectrum of $G_{\times}$must somehow mix those of $G_{A}$, $G_{B}$, and $G$, which is reflected on the corresponding singleparticle entanglement spectra. Unfortunately, however, it is very difficult to separate the various contributions and, in contrast to the case of a single length scale in (91), we have not been able to find a simple ansatz. Nevertheless, from evaluating $\hat{\mathcal{E}}$, we find exactly the same scaling behavior (94) as obtained from CFT. The results are plotted against the proper scaling variable in Fig. 5, finding a perfect collapse of the data. Furthermore, comparing to the result for equal intervals as a function of the segment size, we observe that the two functions match perfectly.

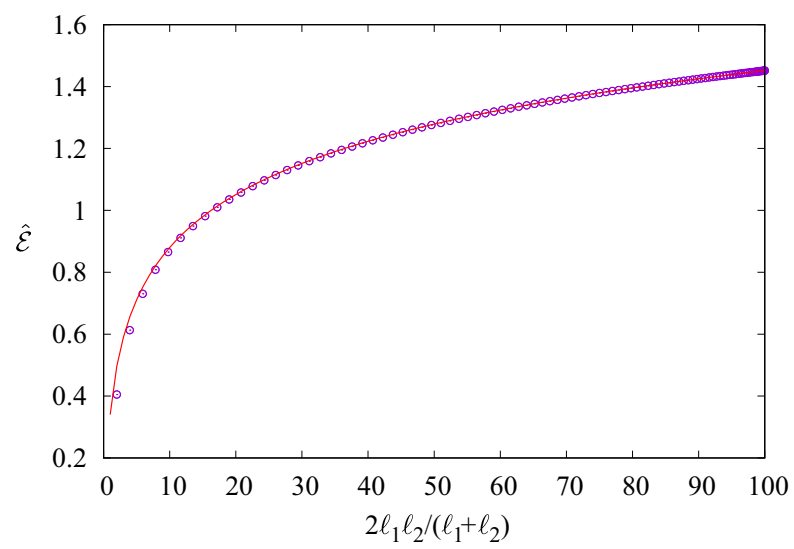

FIG. 5. Upper bound against CFT scaling variable with $\ell_{1}+\ell_{2}=$ 200 fixed and varying $\ell_{1}$. For comparison, the solid line shows the equal-segment result (93), with $\ell_{1}=\ell_{2}=\ell$.

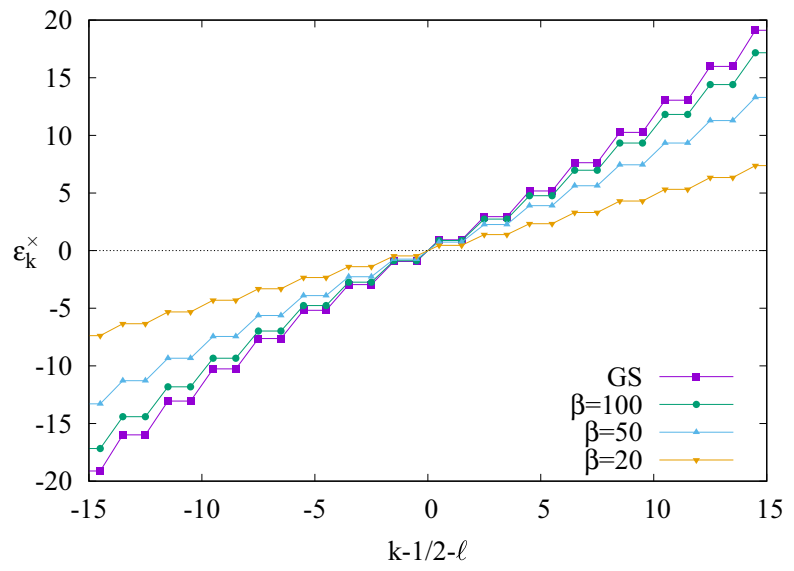

FIG. 6. Thermal single-particle spectra for $\ell=100$ and various $\beta$.

\section{Thermal states}

As our final example, we consider thermal states of the infinite hopping chain with adjacent equal-size segments, where the CFT calculation of the logarithmic negativity gives [24]

$$
\mathcal{E}=\frac{c}{4} \ln \frac{\beta}{\pi} \tanh \frac{\ell \pi}{\beta}+\text { const. }
$$

Hence, for any finite temperatures and $\ell \gg \beta$, the negativity satisfies an area law. To compare it to the behavior of the upper bound, one should first have a look at the corresponding spectra $\varepsilon_{k}^{\times}$, shown in Fig. 6 as a function of $\ell$ and for various $\beta$. One sees the thermal flattening of the spectra with increasing temperatures, which signals a crossover from logarithmic to linear density of states in $\ell$.

As an immediate consequence, the Renyi entropy $S_{1 / 2}\left(\rho_{\times}\right)$ becomes extensive. This, however, does not necessarily spoil the tightness of our upper bound, since the contribution from $S_{2}\left(\rho_{A \cup B}\right)$, which is itself extensive, has to be subtracted. Indeed, as shown in Fig. 7, we find numerically that $\hat{\mathcal{E}}$ saturates for large $\ell$ for any nonzero temperatures and hence the extensive contributions from the two entropies exactly cancel. Moreover, as shown on the inset, we confirm that $\hat{\mathcal{E}}$ has exactly

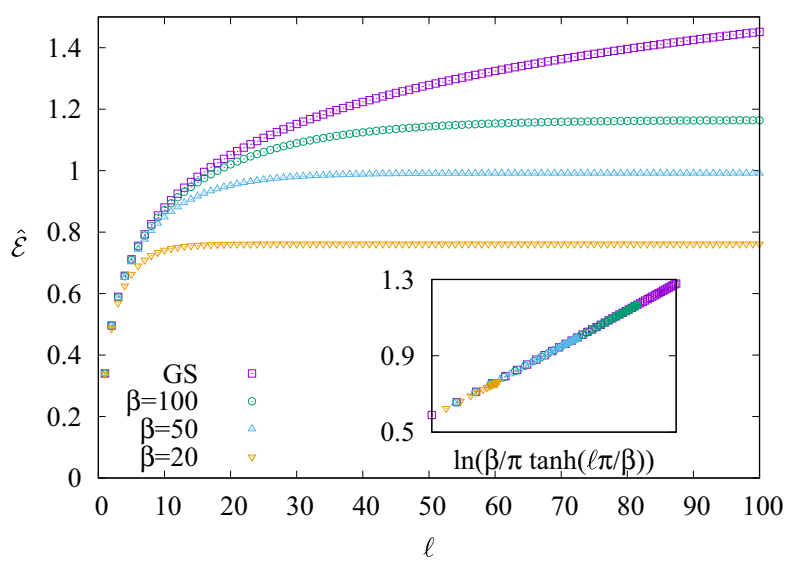

FIG. 7. Upper bound for thermal states with various $\beta$ against $\ell$. The inset shows the data against CFT scaling variable. 
the same scaling behavior as $\mathcal{E}$ in (95). Note, however, that it is difficult to find an analytic argument to understand this type of scaling on the level of the spectra $\varepsilon_{k}^{\times}$, since one has to look for subleading effects.

\section{OUTLOOK}

In conclusion, we have presented rigorous bounds to the entanglement negativity that are efficiently computable for fermionic Gaussian states. In particular, the definition of the lower bound and one of the upper bounds is a simple function of the covariance matrices, allowing an efficient calculation in the number of fermionic modes. Furthermore, we have also constructed an upper bound which makes use of semidefinite programming techniques.

There are a number of questions left open for future research. First, in all our numerical examples, carried out for adjacent intervals in a dimerized hopping chain, we observed that the upper bound of the logarithmic negativity can actually be made more tight by neglecting an additive constant $\ln \sqrt{2}$. Although it has also been conjectured in Ref. [48], we could not give a rigorous proof in support of this claim and it is still unclear if this holds in complete generality.

Moreover, while the upper bound for adjacent intervals in a free-fermion chain gives exactly the same scaling behavior as the CFT prediction for the entanglement negativity, one should also test its performance for the case of nonadjacent intervals. Unfortunately, this setup is much more involved since the analytic continuation from the moments of the partial transpose is not known [60]. Another interesting question is the negativity for nonadjacent intervals in the XX spin chain, where the results in the spin and fermionic basis are not equivalent $[61,62]$, and thus the upper bound should also be properly generalized.
Regarding the lower bound, we observed that it performs particularly well in case of strong singlet-type entanglement between the subsystems. This makes it a good candidate to check the negativity scaling in random singlet phases of disordered spin chains, where the available DMRG results are not yet entirely conclusive [18]. Importantly, the bounds presented here constitute an excellent starting point for endeavors aimed at seeing topological signatures at finite temperatures, as the numerics for comparably small SSH chains already suggests. It is the hope that this work stimulates such further research.

Note added. Upon completion, we became aware of a recent independent work [63], where an alternative definition of fermionic entanglement negativity is considered. Making use of a freedom in the representation of the partial transposition, the authors adopt a different convention, which is equivalent to partial time-reversal. In turn, their entanglement negativity coincides with our upper bound $\hat{\mathcal{E}}$ in Sec. VII.

\section{ACKNOWLEDGMENTS}

We would like to thank discussions and correspondence with Hassan Shapourian, Shinsei Ryu, Ingo Peschel, Christopher Herzog, Yihong Wang, Vladimir Korepin, Erik Tonni, Andrea Coser, and Pasquale Calabrese. J. E. and Z. Z. have been supported by the DFG (CRC183, EI 519/9-1, EI 519/7-1), the Templeton Foundation, and the ERC (TAQ). Z. Z. also acknowledges support from the János Bolyai Research Scholarship of the Hungarian Academy of Sciences and from the Hungarian National Research, Development and Innovation Office (Contracts No. K124152, K124351 and K124176). He would also like to thank the Simons Center for Geometry and Physics for hospitality where some of the work has been carried out. V. E. acknowledges funding from the Austrian Science Fund (FWF) through Projects No. M1854-N36 and No. P30616-N36.
[1] C. H. Bennett, D. P. DiVincenzo, J. A. Smolin, and W. K. Wootters, Phys. Rev. A 54, 3824 (1996).

[2] J. Eisert, M. Cramer, and M. B. Plenio, Rev. Mod. Phys. 82, 277 (2010).

[3] H. Li and F. D. M. Haldane, Phys. Rev. Lett. 101, 010504 (2008).

[4] K. Zyczkowski, P. Horodecki, A. Sanpera, and M. Lewenstein, Phys. Rev. A 58, 883 (1998).

[5] J. Eisert, Ph.D. thesis, University of Potsdam, 2001.

[6] G. Vidal and R. F. Werner, Phys. Rev. A 65, 032314 (2002).

[7] M. B. Plenio, Phys. Rev. Lett. 95, 090503 (2005).

[8] K. M. R. Audenaert, J. Eisert, M. B. Plenio, and R. F. Werner, Phys. Rev. A 66, 042327 (2002).

[9] M. B. Plenio, J. Eisert, J. Dreissig, and M. Cramer, Phys. Rev. Lett. 94, 060503 (2005).

[10] M. Cramer and J. Eisert, New J. Phys. 8, 71 (2006).

[11] M. Cramer, J. Eisert, M. B. Plenio, and J. Dreissig, Phys. Rev. A 73, 012309 (2006).

[12] A. Ferraro, D. Cavalcanti, A. Garcia-Saez, and A. Acin, Phys. Rev. Lett. 100, 080502 (2008).

[13] J. Anders and A. Winter, Quant. Inf. Comp. 8, 0245 (2008).

[14] S. Marcovitch, A. Retzker, M. B. Plenio, and B. Reznik, Phys. Rev. A 80, 012325 (2009).
[15] H. Wichterich, J. Molina-Vilaplana, and S. Bose, Phys. Rev. A 80, 010304(R) (2009).

[16] A. Bayat, P. Sodano, and S. Bose, Phys. Rev. B 81, 064429 (2010).

[17] P. Calabrese, L. Tagliacozzo, and E. Tonni, J. Stat. Mech. (2013) P05002.

[18] P. Ruggiero, V. Alba, and P. Calabrese, Phys. Rev. B 94, 035152 (2016).

[19] V. Alba, J. Stat. Mech. (2013) P05013.

[20] C.-M. Chung, V. Alba, L. Bonnes, P. Chen, and A. M. Läuchli, Phys. Rev. B 90, 064401 (2014).

[21] N. E. Sherman, T. Devakul, M. B. Hastings, and R. R. P. Singh, Phys. Rev. E 93, 022128 (2016).

[22] P. Calabrese, J. Cardy, and E. Tonni, Phys. Rev. Lett. 109, 130502 (2012).

[23] P. Calabrese, J. Cardy, and E. Tonni, J. Stat. Mech. (2013) P02008.

[24] V. Eisler and Z. Zimborás, New J. Phys. 16, 123020 (2014).

[25] P. Calabrese, J. Cardy, and E. Tonni, J. Phys. A 48 (2015).

[26] A. Coser, E. Tonni, and P. Calabrese, J. Stat. Mech. (2014) P12017.

[27] M. Hoogeveen and B. Doyon, Nucl. Phys. B 898, 75 (2015). 
[28] X. Wen, P.-Y. Chang, and S. Ryu, Phys. Rev. B 92, 075109 (2015).

[29] O. Blondeau-Fournier, O. A. Castro-Alvaredo, and B. Doyon, J. Phys. A 49, 125401 (2016).

[30] H. Wichterich, J. Vidal, and S. Bose, Phys. Rev. A 81, 032311 (2010).

[31] R. A. Santos, V. Korepin, and S. Bose, Phys. Rev. A 84, 062307 (2011).

[32] R. A. Santos and V. Korepin, Quantum Inf. Process. 15, 4581 (2016).

[33] P.-Y. Chang and X. Wen, Phys. Rev. B 93, 195140 (2016).

[34] V. Eisler and Z. Zimborás, Phys. Rev. B 93, 115148 (2016).

[35] C. De Nobili, A. Coser, and E. Tonni, J. Stat. Mech. (2016) 083102.

[36] Y. A. Lee and G. Vidal, Phys. Rev. A 88, 042318 (2013).

[37] C. Castelnovo, Phys. Rev. A 88, 042319 (2013).

[38] X. Wen, P.-Y. Chang, and S. Ryu, JHEP 09 (2016) 012.

[39] X. Wen, S. Matsuura, and S. Ryu, Phys. Rev. B 93, 245140 (2016).

[40] J. Eisert and M. B. Plenio, J. Mod. Opt. 46, 145 (1999).

[41] J. Eisert and M. B. Plenio, Int. J. Quant. Inf. 1, 479 (2003).

[42] G. Adesso and F. Illuminati, J. Phys. A 40, 7821 (2007).

[43] A. Peres, Phys. Rev. Lett. 77, 1413 (1996).

[44] R. Simon, Phys. Rev. Lett. 84, 2726 (2000).

[45] V. Eisler and Z. Zimborás, New J. Phys. 17, 053048 (2015).

[46] A. Y. Kitaev, Ann. Phys. (NY) 321, 2 (2006).
[47] A. J. Heeger, S. Kivelson, J. R. Schrieffer, and W. P. Su, Rev. Mod. Phys. 60, 781 (1988).

[48] C. P. Herzog and Y. Wang, J. Stat. Mech. (2016) 073102.

[49] A. Botero and B. Reznik, Phys. Lett. A 331, 39 (2004).

[50] J. I. Latorre, E. Rico, and G. Vidal, Quant. Inf. Comput. 4, 48 (2004).

[51] B. Q. Jin and V. E. Korepin, J. Stat. Phys. 116, 79 (2004).

[52] S. Bravyi, Quantum Inf. Comput. 5, 216 (2005).

[53] J. Eisert, S. Scheel, and M. B. Plenio, Phys. Rev. Lett. 89, 137903 (2002).

[54] G. Giedke and J. I. Cirac, Phys. Rev. A 66, 032316 (2002).

[55] R. Bhatia, Matrix Analysis (Springer, New York, 1997).

[56] M. Fagotti and P. Calabrese, J. Stat. Mech. (2010) P04016.

[57] I. Peschel and V. Eisler, J. Phys. A: Math. Theor. 42, 504003 (2009).

[58] I. Peschel, J. Stat. Mech. (2004) P06004.

[59] V. Eisler and I. Peschel, J. Stat. Mech. (2013) P04028.

[60] A. Coser, E. Tonni, and P. Calabrese, J. Stat. Mech. (2016) 033116.

[61] A. Coser, E. Tonni, and P. Calabrese, J. Stat. Mech (2015) P08005.

[62] A. Coser, E. Tonni, and P. Calabrese, J. Stat. Mech (2016) 053109.

[63] H. Shapourian, K. Shiozaki, and S. Ryu, Phys. Rev. B 95, 165101 (2017). 